\title{
Pincement de l'interligne entre processus coronoïde de la mandibule et procès alvéolaire du maxillaire. A propos d'un cas
}

\author{
Fauroux MA, Torres JH \\ Service d'Odontologie, CHU, Montpellier, France \\ alix.et.jp@free.fr
}

Peu d'anomalies du processus coronoïde de la mandibule sont répertoriées dans la littérature (Goudot et al. 1989, Zhong et al. 2009). On retrouve essentiellement une série de cas (Escuder et al 2001) dont le diagnostic est établi sur des anomalies de forme du processus coronoïde, s'intégrant dans le syndrome de Jacob (interférence entre le processus coronoïde et l'arcade zygomatique), associé ou non à une maladie de Langenbeck (hyperplasie du processus coronoïde).

Un homme âgé de 35 ans consulte pour des douleurs maxillaires droites irradiant vers la tubérosité. Selon le patient, ces douleurs se manifestent depuis un an environ, et seraient apparues à la suite de l'avulsion de la dent de sagesse maxillaire homolatérale. Elles sont ressenties de façon quasi-constante, mais le patient rapporte deux épisodes particulièrement violents ces derniers mois.

L'examen clinique régional ne montre rien de particulier : la palpation exobuccale est normale et non douloureuse ; il n'y a pas de limitation de l'ouverture buccale ni de latérodéviation ; il n’y a pas d'asymétrie faciale notable ; la muqueuse buccale est saine ; l'écoulement salivaire est clair; il n’y a pas d'adénopathies cervicales. On ne retrouve pas d'antécédent de traumatisme.

Il ne ressort seulement de l'examen clinique qu'une très importante asymétrie du défilé entre le processus coronoïde de la mandibule d'une part et le rempart alvéolaire vestibulaire du maxillaire d'autre part : environ un doigt et demi du côté gauche pour quelques millimètres à peine du côté droit où une exostose alvéolaire réalise une saillie longitudinale horizontale. L'orthopantomogramme n'apporte aucune contribution ; une tomodensitométrie est demandée pour illustrer en trois dimensions le rapport entre les pièces osseuses.

Des explications sont données au patient ainsi que des conseils pour éviter les situations d'attrition des tissus mous, en particulier la recommandation d'arrêter la consommation de chewing-gum. Le patient est rassuré. Mais souhaitant en outre éviter tout nouvel épisode hyperalgique, il est demandeur d'une solution radicale. Dans un second temps, si les douleurs persistent, une résection chirurgicale de l'exostose alvéolaire lui sera donc proposée.

Dans cette situation clinique exceptionnelle, l'origine mécanique des douleurs semble évidente du fait de l'interférence entre les pièces osseuses lors de la fonction : la douleur ressentie par le patient peut être expliquée par le frottement permanent du processus coronoïde droit contre l'os maxillaire, qui pince la muqueuse au cours des mouvements mandibulaires, en particulier en diduction gauche. Il ne s'agit donc pas d'un cas de syndrome de Jacob, dans lequel les douleurs sont inconstantes et où la «néo-articulation » se situe entre le processus coronoïde d'une part et le malaire d'autre part, et non pas comme ici entre le processus coronoïde et les procès alvéolaires des molaires maxillaires. 